\title{
Influencia de la especialización del cirujano en los resultados tras cirugía por cáncer de colon. Utilidad de los índices de propensión (propensity scores)
}

\author{
D. Martínez-Ramos, J. Escrig-Sos, J. M. Miralles-Tena, M. I. Rivadulla-Serrano, J. M. Daroca-José \\ y J. L. Salvador Sanchís
}

Servicio de Cirugía General y Digestiva. Hospital General de Castellón

\section{RESUMEN}

Introducción: la influencia del factor cirujano en el cáncer colorrectal se ha estudiado de manera reiterada en literatura científi$\mathrm{ca}$, pero los resultados han sido contradictorios. Se plantea en este estudio si la especialización del cirujano es un factor determinante del pronóstico en estos pacientes. Asimismo, se valora la importancia de los índices de propensión (PS) en el análisis de los resultados.

Pacientes y métodos: se planteó un estudio retrospectivo con revisión de las historias clínicas de 236 pacientes sometidos a cirugía por cáncer de colon en el Hospital General de Castellón. Se establecieron dos grupos de cirujanos (especialista y no especialista), comparando los resultados en cuanto a supervivencia y en cuanto a intervalo libre de enfermedad a los 5 años. Inicialmente, se realizaron las comparaciones sin el ajuste de los resultados y, posteriormente, utilizando los PS.

Resultados: el primer análisis de los resultados (sin ajuste) fue claramente favorable al grupo de cirujanos especialistas (supervivencia a los 5 años del 64,3 frente al 79,3\%, p =0,028). No obstante, cuando se ajustó mediante los PS, no se obtuvo dicha significación estadística.

Conclusiones: no se ha podido demostrar que la especialización del cirujano sea un factor determinante del pronóstico en los pacientes sometidos a cirugía por cáncer de colon. Los índices de propensión pueden ser de gran utilidad para el ajuste de los resultados en estudios retrospectivos no aleatorizados, especialmente cuando el factor de estudio es poco frecuente.

Palabras clave: Cáncer de colon. Resultados. Especialización. Índices de propensión.

\begin{abstract}
Introduction: surgeon influence on colorectal cancer surgery outcomes has been repeatedly studied in the scientific literature, but conclusions have been contradictory. Here we study whether surgeon specialization is a determinant factor for outcome in these patients. The importance of propensity scores (PS) in surgical research is also studied.

Patients and methods: a retrospective study was performed and medical records were reviewed for 236 patients who were intervened for colon cancer in Castellon General Hospital (Spain). Cases were divided into two groups (specialist and non-specialist surgeons), and both 5-year surveillance and disease free survival were compared. Comparisons were first made with no adjustments, and then subsequently using PS analysis.

Results: the initial (non-adjusted) analysis was clearly favourable for the specialist surgeon group (5-year surveillance, $64.3 \mathrm{vs}$. $79.3 \%, p=0.028$ ). After adjusting for PS no statistical significance was obtained.

Conclusions: surgeon specialization had no significant impact on patient outcome after colon cancer surgery. Propensity score analysis is an important tool in the analysis of surgical non-randomized studies, particularly when events under scrutiny are rare.
\end{abstract}

Key words: Colon cancer. Outcomes. Specialization. Propensity scores.

Martínez-Ramos D, Escrig-Sos J, Miralles-Tena JM, Rivadulla-Serrano MI, Daroca-José JM, Salvador Sanchís JL. Influencia de la especialización del cirujano en los resultados tras cirugía por cáncer de colon. Utilidad de los índices de propensión (propensity scores). Rev Esp Enferm Dig 2008; 100: 387-392.

Recibido: 03-12-07.

Aceptado: 17-03-08.

Correspondencia: David Martínez-Ramos. Servicio de Cirugía General y Digestiva. Hospital General de Castellón. Avda. Benicàssim, s/n. 12004 Castellón.e-mail: davidmartinez@comcas.es

\section{INTRODUCCIÓN}

La influencia del factor cirujano en los resultados quirúrgicos ha sido analizada de manera reiterada y para diferentes tipos de cáncer en la literatura científica mundial $(1,2)$. El cáncer de colon no ha sido una excepción y mu- 
chos autores han estudiado la posible relación entre el grado de especialización en cirugía colorrectal de un cirujano y sus resultados (3-20). No obstante, estos estudios comparativos (cirujano especialista frente a cirujano no especialista) no siempre han contado con las garantías metodológicas necesarias para extraer conclusiones basadas en la auténtica evidencia científica. De este modo, si bien es cierto que los ensayos clínicos prospectivos y aleatorizados eliminan los sesgos debidos a las características biológicas de cada paciente y de su tumor (edad, sexo, estadio tumoral, comorbilidad, etc.), al distribuirlos de forma equilibrada en ambos grupos de estudio, en los estudios retrospectivos no es posible asegurar que esta distribución sea realmente aleatoria. Dicho de otro modo, en los estudios no aleatorizados pueden existir importantes desigualdades en la distribución de las características propias de cada tumor y de su huésped y esta distribución no aleatoria puede condicionar y falsear finalmente el resultado del estudio.

Para disminuir la influencia de los factores de confusión en los análisis no aleatorizados, Rosenbaum y Rubin (21) introdujeron, en 1983, el concepto de propensity score (PS), también llamado índice de propensión, score de propensión o puntaje de propensión. Este índice es un valor comprendido entre 0 y 1 , que indica la probabilidad de que un paciente (con unas determinadas características biológicas) recaiga en una rama del estudio o en otra, cuando no ha habido aleatorización $(22,23)$. Cuando los resultados se ajustan utilizando esta probabilidad, aunque con ciertas limitaciones, puede asumirse que los rasgos de los pacientes de estudio que puedan tener influencia en el resultado se distribuirán de una manera "casi-aleatoria".

El objetivo del presente estudio fue analizar la influencia del factor cirujano (especialista frente a no especialista) en los resultados del cáncer de colon, así como poner de manifiesto la importancia de los PS en este tipo de análisis comparativo.

\section{MATERIAL Y MÉTODOS}

Para cubrir los objetivos del estudio, se utilizaron los datos de 236 pacientes intervenidos de cáncer de colon en el Hospital General de Castellón, entre los años 1995 y 2000, sometidos a resección tumoral tanto con intención curativa como paliativa. Se excluyeron, pues, todas las intervenciones sin resección del tumor primario, así como los casos de cáncer de recto. El objetivo de elegir este periodo de estudio fue conseguir un periodo de seguimiento mínimo de 5 años.

Para la confección del PS se utilizó la regresión logística, en la que la variable de resultado fue el tipo de cirujano. Como variables de predicción se manejaron aquellas de nuestra base de datos que, de alguna forma, pudieran influir teóricamente en el resultado del tratamiento quirúrgico y, consecuentemente, de la enfermedad. Asimismo, estas variables no debían depender de la elección o actuación del cirujano, ni corresponder al resultado del caso, una vez pro- ducido este, en cuanto a supervivencia e intervalo libre de enfermedad, ya que tanto la supervivencia como el intervalo libre de enfermedad constituyeron los objetivos principales del análisis. Se trata de variables referidas a los pacientes, tales como edad y comorbilidad grave, referidas a la posible dificultad de la intervención, como la localización tumoral y, por último, referidas a los rasgos basales del tumor, como el estadio de Dukes y el grado histológico. Se pidió al programa estadístico que guardara en la base de datos una nueva variable consistente en la probabilidad derivada del modelo logístico, es decir, la probabilidad, dadas estas variables de predicción, de que cada caso fuera tratado por el cirujano correspondiente. En este estudio, dicha probabilidad constituye el PS.

Como comorbilidad grave se entendió la presencia previa de enfermedades de riesgo en tratamiento en el momento de la cirugía: cardiopatía, hepatopatía, insuficiencia renal, diabetes, enfermedad pulmonar crónica y accidente vascular periférico. Se definieron como del lado derecho los tumores primarios localizados entre el ciego y los dos tercios proximales del colon transverso; y como del lado izquierdo los tumores primarios localizados entre el tercio distal del colon transverso y la zona inmediatamente proximal a la unión rectosigmoidea. Los estadios de Dukes comprendían los clásicos A, B y C, a los que se añadió un grupo, denominado $\mathrm{D}$, para incluir los casos con metástasis sincrónicas con la cirugía, que en todos los casos afectaban al hígado.

Se consideró como cirujanos especialistas aquellos pertenecientes a la Sección de Coloproctología (3 cirujanos) y como no especialistas a los restantes cirujanos del servicio que habían realizado alguna intervención por cáncer de colon (8 cirujanos). En nuestro servicio, no hay ningún canal formal de distribución preestablecido para el reparto entre ambos grupos de la cirugía electiva del cáncer de colon en concreto.

Se analizaron, en primer lugar, las variables correspondientes a dichas características basales, tanto de los pacientes como del tumor, así como los resultados del tratamiento para supervivencia general de la enfermedad y para el intervalo libre de recidiva, sin ningún tipo de ajuste, de acuerdo con ambos grupos de cirujanos. Las variables cuantitativas se describen con su media \pm desviación estándar y las cualitativas con su frecuencia y porcentaje relativo. Se utilizó la prueba de la t de Student para variables cuantitativas, la prueba exacta de Fisher para las variables cualitativas y el método de Kaplan-Meier con la prueba del log-rank para analizar supervivencias.

Posteriormente, se analizó la supervivencia general y el intervalo libre de enfermedad (tiempo de aparición de recidiva de la enfermedad en aquellos casos sin metástasis iniciales diagnosticadas) mediante regresión de Cox. En esta prueba multivariante únicamente se incluyeron como variables de predicción el tipo de cirujano y la puntuación PS como elemento de ajuste de la anterior. Se utilizó el programa estadístico STATA versión 9. El corte de significación estadística fue el habitual de 0,05. 


\section{RESULTADOS}

De los 236 pacientes incluidos en el estudio, $127(53,8 \%)$ fueron varones y $109(46,2 \%)$ fueron mujeres. La media de edad fue de $67,7 \pm 12$ años, con un mínimo de 30 años y un máximo de 92 años. El tumor se localizó en 154 ocasiones $(65,3 \%)$ en el lado izquierdo del colon, localizándose en 82 ocasiones $(34,7 \%)$ en el lado derecho. En 148 pacientes $(62,7 \%)$ se identificó algún tipo de comorbilidad previa al acto quirúrgico. La distribución por estadios de Dukes fue la siguiente: estadio A, 31 pacientes (13\%); estadio B, 105 pacientes (44\%); estadio C, 72 pacientes (31\%); y estadio D, 28 pacientes (12\%). El grado histológico del tumor fue: GX, 2 pacientes; G1, 140 pacientes $(59,3 \%)$; G2, 85 pacientes (36\%); y G3, 9 pacientes (4\%). El tiempo anestésico medio fue de $161,4 \pm 51$ minutos. En 97 pacientes (41\%) se encontró algún ganglio positivo, siendo la media de ganglios linfáticos analizados por paciente de 10,4 \pm 7 ganglios. En 191 pacientes $(80,9 \%)$ no se presentaron complicaciones en el postoperatorio inmediato, apareciendo, en 6 ocasiones, infección de la herida quirúrgica; en 3 ocasiones evisceración; en 4 ocasiones fallo de sutura; en 18 ocasiones otro tipo de complicaciones; y en 14 ocasiones $(6 \%)$ la muerte del paciente. En cuanto al seguimiento, la supervivencia global a los 5 años fue del $64,1 \%$, produciéndose la muerte por tumor en 72 ocasiones $(31 \%)$. La supervivencia global libre de enfermedad a los 5 años fue del $71,5 \%$.

El grupo de cirujanos especialistas realizó un total de 111 intervenciones, lo que supuso un promedio de 7,4 intervenciones al año por cada cirujano, mientras que el grupo de no especialistas realizó un total de 125 intervenciones (3 intervenciones/año/cirujano). En las tablas I y II se muestran los resultados obtenidos tras el análisis compara-

Tabla I. Características basales de los pacientes y del tumor

\begin{tabular}{lccc}
\hline & $\begin{array}{r}\text { Cirujano no } \\
\text { especialista }\end{array}$ & $\begin{array}{c}\text { Cirujano } \\
\text { especialista }\end{array}$ & $\begin{array}{c}\text { Valor } \\
p\end{array}$ \\
\hline $\begin{array}{l}\text { Número de pacientes } \\
\text { Edad }\end{array}$ & 125 & 111 & - \\
Comorbilidad & $67,9 \pm 12$ & $67,4 \pm 12$ & $0,782^{*}$ \\
Sí & $77(52 \%)$ & $71(48 \%)$ & $0,788 * *$ \\
No & $48(55 \%)$ & $40(45 \%)$ & \\
Lado del tumor & & & \\
Derecho & $40(49 \%)$ & $42(51 \%)$ & $0,411 * *$ \\
Izquierdo & $85(55 \%)$ & $69(45 \%)$ & \\
Estadio de Dukes & $18(58 \%)$ & $13(42 \%)$ & \\
A & $59(56 \%)$ & $46(44 \%)$ & $0,049 * *$ \\
B & $29(40 \%)$ & $43(60 \%)$ & \\
C & $19(68 \%)$ & $9(32 \%)$ & \\
D & & & \\
Grado histológico & 2 & 0 & \\
No determinado & $74(53 \%)$ & $66(47 \%)$ & $0,710 * *$ \\
G1 (bien diferenciado) & $45(53 \%)$ & $40(47 \%)$ & \\
G2 (intermedio) & $4(44 \%)$ & $5(56 \%)$ & \\
G3 (mal diferenciado) & & \\
\hline
\end{tabular}

'Expresado con media \pm desviación estándar; *Prueba de la t de Student; **Prueba exacta de Fisher. tivo entre ambos grupos, pero sin ajustar la distribución de los pacientes. De este estudio se desprende que los únicos factores que alcanzaron la significación estadística fueron el estadio de Dukes (más metástasis sincrónicas en el grupo de cirujanos no especialistas y más casos con ganglios positivos en los especialistas) y la supervivencia global a los 5 años, que fue mayor en el grupo de cirujanos especialistas (Fig. 1). Es decir, cuando no se ajustaron los resultados, el grupo de cirujanos especialistas obtuvo mejores resultados en cuanto a probabilidad de supervivencia a los 5

Tabla II. Resultados del tratamiento quirúrgico previos al ajuste mediante propensity scores

\begin{tabular}{|c|c|c|c|}
\hline & $\begin{array}{l}\text { Cirujano no } \\
\text { especialista }\end{array}$ & $\begin{array}{c}\text { Cirujano } \\
\text { especialista }\end{array}$ & $\begin{array}{l}\text { Valor } \\
p\end{array}$ \\
\hline Número de pacientes & 125 & 111 & - \\
\hline Tiempo anestésico (minutos) ${ }^{\dagger}$ & $162,3 \pm 53$ & $160,4 \pm 50$ & $0,772^{*}$ \\
\hline Ganglios analizados ${ }^{\dagger}$ & $9,6 \pm 7$ & $11,3 \pm 8$ & $0,063^{*}$ \\
\hline Ganglios positivos $^{\dagger}$ & $1,2 \pm 3$ & $1,5 \pm 2$ & $0,416^{*}$ \\
\hline \multicolumn{4}{|l|}{ Complicaciones postoperatorias } \\
\hline No & $101(81 \%)$ & $90(81 \%)$ & \\
\hline Infección herida & $1(1 \%)$ & $5(4 \%)$ & \\
\hline Evisceración & $1(1 \%)$ & $(2 \%)$ & $0,078^{* *}$ \\
\hline Fallo sutura (§) & $4(3 \%)$ & 0 & \\
\hline Exitus (§§) & $10(8 \%)$ & $4(4 \%)$ & \\
\hline Otras & $8(6 \%)$ & $10(9 \%)$ & \\
\hline \multicolumn{4}{|l|}{ Muerte por el tumor } \\
\hline No & $79(48 \%)$ & $85(52 \%)$ & \\
\hline Sí & $46(64 \%)$ & $26(36 \%)$ & \\
\hline Supervivencia, 5 años (Kaplan-Meier) & $64,3 \%$ & $79,3 \%$ & $0,028^{* * *}$ \\
\hline \multicolumn{4}{|l|}{ Recidiva del tumor } \\
\hline No & $102(56 \%)$ & $79(43 \%)$ & \\
\hline Sí & $23(42 \%)$ & $32(58 \%)$ & \\
\hline \multicolumn{4}{|l|}{ Supervivencia libre de enfermedad, } \\
\hline 5 años (Kaplan-Meier) & $77,8 \%$ & $75,4 \%$ & $0,197^{\star \star \star}$ \\
\hline
\end{tabular}

${ }^{\top}$ Expresado con media \pm desviación estándar; (§): particular para fallo de sutura, $\mathrm{p}$ $=0,177 * * ;(\S \S)$ : particular para exitus, $p=0,125^{* *}$; *Prueba de la t de Student;

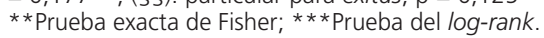

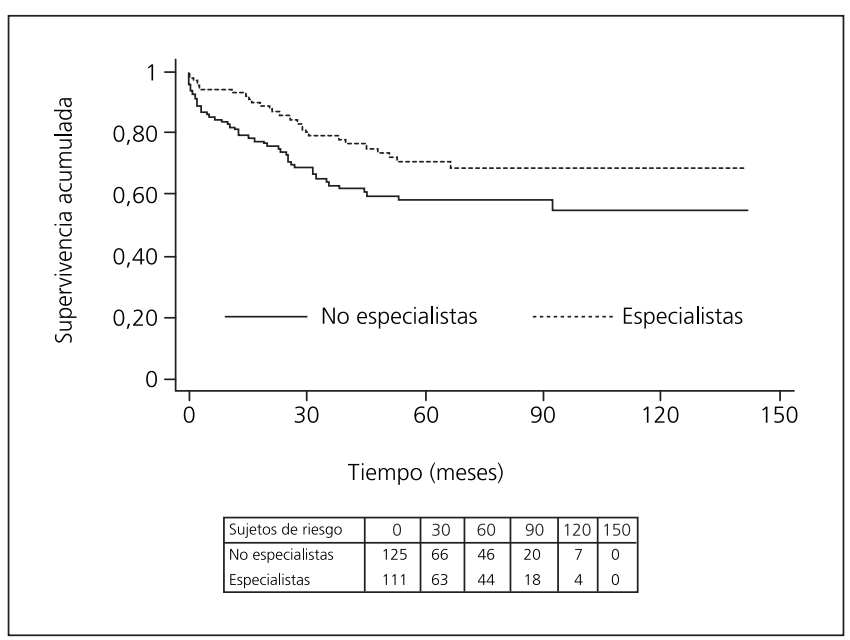

Fig. 1. Probabilidad de supervivencia según el método de Kaplan-Meier para cirujanos especialistas y para cirujanos no especialistas. Resultados previos a su ajuste. 
años, con una diferencia del 15\% (64,3 frente a 79,3\%). Otros factores, como el número de ganglios analizados y las complicaciones postoperatorias, rozaron esta significación estadística, lo que podría ser interpretado como una cierta tendencia en el grupo de cirujanos especialistas a obtener mayor número de ganglios y distintas complicaciones postoperatorias. En este análisis no se demostraron diferencias en la supervivencia libre de enfermedad en ambos grupos de estudio.

A pesar de estos resultados, claramente favorables al grupo de cirujanos especialistas, cuando se realizó el ajuste mediante PS dentro de la regresión de Cox para la supervivencia y para la recidiva de la enfermedad, se obtuvieron los resultados que se muestran en la tabla III. De este modo, tras el ajuste, se observa que el valor de p para el PS en la recidiva tumoral fue cercano a $1(p=0,92)$, esto es, claramente no significativo, mientras que en el caso de la supervivencia, este fue inferior a 0,001 , altamente significativo. Dicho de otro modo, la distribución de los pacientes entre ambos tipos de cirujanos influyó muy poco en el resultado obtenido para la recidiva, pero, al contrario, esta distribución fue la que realmente condicionó las diferencias en la supervivencia global. Así, las diferencias obtenidas previamente mediante el análisis sin ajuste de los resultados no serían atribuibles al grado de especialización del cirujano sino a la diferente probabilidad de los pacientes de ser asignados a uno u otro grupo de cirujanos según sus propias características basales (edad, comorbilidad, lado del tumor, estadio de Dukes y grado histológico, agrupadas conjuntamente en el PS). En definitiva, no se ha podido demostrar que el factor cirujano, en cuanto a su especialización, influya en la supervivencia general y en el intervalo libre de enfermedad en la cirugía del cáncer de colon en nuestros pacientes.

\begin{tabular}{|c|c|c|c|}
\hline & $\begin{array}{l}\text { Hazard } \\
\text { ratio }\end{array}$ & $\begin{array}{c}\text { Intervalo de } \\
\text { confianza al 95\% }\end{array}$ & $\begin{array}{c}\text { Valor } \\
p\end{array}$ \\
\hline \multicolumn{4}{|l|}{ Recidiva tumoral } \\
\hline Cirujano especialista & 1,43 & 0,83 a 2,46 & $0,201 *$ \\
\hline $\begin{array}{l}\text { Propensity score } \\
\text { Supervivencia }\end{array}$ & - & - & $0,921 *$ \\
\hline Cirujano especialista & 0,74 & 0,46 a 1,20 & $0,224 *$ \\
\hline Propensity score & - & - & $<0,001 * *$ \\
\hline
\end{tabular}

\section{DISCUSIÓN}

El grado de especialización del cirujano se ha demostrado como un factor pronóstico independiente para diferentes tipos de cirugía compleja y poco frecuente, tales como la pancreatectomía, la esofaguectomía, las neumo- nectomías, las resecciones hepáticas o las exanteraciones pélvicas (1). En el cáncer colorrectal, sin embargo, los resultados son contradictorios. De este modo, McArdle y Hole $(10)$, entre otros $(7,20)$, demostraron que los cirujanos con mayor experiencia o especialistas en cirugía colorrectal obtenían mejores resultados en cuanto a supervivencia y en cuanto a recidiva que los no especialistas. Resultados similares obtuvieron Harmon y cols. (5) y Schrag y cols. $(17,19)$, aunque, a diferencia de lo propuesto por McArdle y Hole (10), estos autores afirmaron que el volumen de pacientes atendidos en el hospital sería un factor más potente que el propio grado de especialización de cada cirujano. No obstante, aunque estadísticamente significativa, la diferencia en la mortalidad postoperatoria tras cirugía por cáncer de colon entre los hospitales con mayor y menor volumen de pacientes fue únicamente del 2\% (16). Estos resultados contrastan con los obtenidos por Hermanek y cols. (6), quienes obtuvieron una tasa de recidiva local similar entre cirujanos con menos de 15 procedimientos quirúrgicos sobre el recto y los cirujanos con un volumen de pacientes mayor. Otros dos estudios $(8,13)$ tampoco fueron capaces de demostrar una relación estadísticamente significativa entre el número de casos por cirujano y la supervivencia. El trabajo de Mella y cols. (11) sobre 3.221 pacientes con cáncer colorrectal tampoco consiguió demostrar diferencias en la supervivencia postoperatoria entre un grupo de cirujanos autodenominados expertos y otro grupo considerado no experto. Simunovic y cols. (19) no encontraron diferencias en la supervivencia de los pacientes sometidos a cirugía por cáncer específicamente de colon en Ontario (Canadá) en los hospitales con mayor o menor volumen de pacientes operados.

En este contexto, el presente estudio no fue capaz de demostrar diferencias significativas desde el punto de vista estadístico en cuanto a la supervivencia global a los 5 años ni en la supervivencia libre de enfermedad entre el grupo de cirujanos especialistas y el grupo de cirujanos no especialistas. No obstante, la principal limitación del presente trabajo radica en la definición del concepto de cirujano especialista en cirugía colorrectal ya que, en el momento actual, no existen criterios nacionales ni internacionales que definan este concepto o el de especialista en cirugía oncológica del colon. En ocasiones, para calificar a un cirujano como especialista se ha utilizado el volumen de pacientes intervenidos por cirujano (9) o por hospital (18), la pertenencia a alguna asociación específica de coloproctología $(16,20)$, los años como especialista (7), la posesión del título de board en coloproctología $(14,15)$ o, incluso, la autodenominación de especialista con interés especial por la coloproctología (11). En otras ocasiones, como en el presente trabajo, la distinción entre cirujano especialista o no especialista se establece por la asignación de los investigadores en uno u otro grupo (peer assessment), según ciertos criterios establecidos previamente (10). En nuestro estudio, esta asignación fue exclusivamente por la pertenencia o no a la Sección de 
Coloproctología de nuestro Servicio de Cirugía General. Aunque, lógicamente, es necesario establecer y definir qué se entiende por cirujano especialista en coloproctología, conviene tener en cuenta las limitaciones que estas diferencias de concepto puedan representar para el análisis comparativo de los resultados. Otra diferencia que conviene tener presente es que el actual estudio hace referencia única y exclusivamente al cáncer de colon, siendo excluidos los cánceres localizados en el recto. Como es sabido, los cánceres rectales presentan importantes diferencias en cuanto a dificultad técnica y en cuanto a tratamientos adyuvantes y neoadyuvantes con respecto a los cánceres localizados en el colon, por lo que los resultados actuales no pueden ser aplicados a los tumores de recto.

Por otro lado, el presente trabajo ha demostrado, de una manera sencilla, la utilidad de los índices conocidos como propensity scores, pues los resultados que, en principio y dentro de un análisis crudo sin ajustar por posibles factores de confusión, eran claramente favorables al grupo de especialistas (supervivencia a los 5 años del 64,3 vs. $79,3 \%, p=0,028$ ) perdieron la significación estadística tras aplicar este método de análisis. Según este análisis, determinados pacientes tuvieron mayor probabilidad, a priori, de ser asignados a un grupo o a otro, lo que producía un importante sesgo en los primeros resultados. Una vez hecho el ajuste utilizando los PS, no pudo demostrarse que el factor cirujano tuviera importancia sobre el pronóstico en los pacientes sometidos a cirugía en el cáncer de colon. Una leve tendencia no significativa que se mantenía tras el ajuste, hacia una mejor supervivencia a favor del grupo de especialistas (hazard ratio < 1) (Tabla III), podría quizá tener relación con que operaron menos casos que de entrada presentaban metástasis. Por el contrario, el grupo de especialistas mostró una leve tendencia desfavorable, de nuevo estadísticamente no significativa (hazard ratio $>1$ ) (Tabla III), en cuanto al intervalo hasta la recidiva, quizá explicable porque operaron más casos con ganglios positivos (Dukes C) y menos casos en estadios A y B. Aun así, una ventaja de los PS es que también recogen el peso, por pequeño que pudiera ser, de las variables que individualmente no muestran diferencias significativas con respecto a los grupos sometidos a comparación.

Si bien es cierto que la comparación de los resultados quirúrgicos es una de las herramientas necesarias para poder realizar una cirugía basada en la evidencia, no es menos cierto que estas comparaciones deben ser hechas con cierto rigor pues, de lo contrario, las conclusiones obtenidas podrían ser sesgadas y sus consecuencias terribles. De este modo, cuando estas comparaciones se realizan en el seno de ensayos clínicos controlados y aleatorizados, los pacientes son distribuidos al azar en los diferentes grupos o ramas del estudio y puede asumirse, por tanto, que las diferencias entre ambos grupos son mínimas en cuanto a factores de confusión. No obstante, estos estudios no siempre pueden realizarse de forma prospectiva y aleatorizada por diferentes motivos, especialmente éticos y or- ganizativos, debiendo recurrirse, como en el presente trabajo, a estudios de series de casos, de cohortes o de casocontrol. En estos estudios no aleatorizados, en general, los factores de confusión son un importante problema y el análisis multivariante es utilizado de manera frecuente para su ajuste. Sin embargo, en ocasiones, este tipo de análisis, aplicado directamente al objetivo del estudio, puede estar excesivamente forzado en su capacidad, especialmente cuando el resultado de estudio es raro (por ejemplo, muerte en pacientes con tumores en estadios iniciales) o, lo que es igual, cuando el número de factores de confusión es demasiado elevado para la muestra empleada. En estos casos, los PS ofrecen una alternativa válida desde el punto de vista matemático al análisis multivariante directo $(22,23)$. Así, los PS representan la probabilidad (entre 0 y 1 ) de que un paciente en concreto reciba un tratamiento en lugar del tratamiento del otro grupo control, es decir, de ser "asignado" a un grupo de estudio, dadas unas características basales observadas (factores de confusión en potencia), utilizando para ello un modelo de regresión logística como paso intermedio al análisis definitivo. La principal diferencia entre el análisis multivariante directo y los PS radica en que en el análisis multivariante directo se ajusta de acuerdo a unas características de base, prestando especial atención a la relación entre estas características y el resultado final, mientras que los PS se centran en la relación entre las características de base y la variable principal objeto de estudio, en nuestro caso el tipo de cirujano, para, en un segundo paso, ser utilizado como elemento de ajuste en el análisis final de los objetivos principales. De este modo, los PS intentan reconstruir, a posteriori, una situación similar a la aleatorización, denominada casi-aleatorización (quasi-randomization) (22). Las técnicas analíticas más frecuentemente utilizadas para el cálculo de los PS en medicina son el emparejamiento, la estratificación y la regresión logística. En el presente estudio se utilizó esta última, por su mayor sencillez en su aplicación sobre las otras modalidades y se incluyeron, de modo intencionado, como potenciales factores de confusión la edad, la comorbilidad, el lado del tumor, el estadio tumoral y el grado histológico, factores habitualmente usados en el ajuste de resultados quirúrgicos en esta enfermedad (16). No obstante, es importante recordar que los PS, igual que las técnicas de análisis multivariante usadas de forma directa, no permiten el ajuste por factores de confusión desconocidos o no disponibles y, de existir uno de estos factores desconocidos o no recogidos, podría alterarse el resultado final (22). Esto es otra limitación del estudio, pero debida exclusivamente al propio método utilizado.

La utilidad de los PS va mucho más allá de la aquí presentada para la comparación de resultados entre cirujanos especialistas y cirujanos no especialistas, ya que pueden ser utilizados para la comparación de los resultados en cualquier ámbito, tales como dos técnicas quirúrgicas (laparoscópica frente a abierta), dos tratamientos adyuvantes (quimioterapia adyuvante frente a quimioterapia neoadyuvante), dos centros hospitalarios (de referencia 
frente a comarcal), etc. Por ello, conocer sus indicaciones, su mayor o menor facilidad de aplicación, sus consecuencias y sus limitaciones, puede ser de gran ayuda para evitar la posibilidad de importantes errores en las conclusiones de nuestros estudios. En general, a la hora de comparar dos grupos en estudios no aleatorizados, es necesario establecer algún tipo de ajuste según posibles factores de confusión. No es suficiente, ni ofrece suficientes garantías, analizar la distribución de estos factores entre ambos grupos y no encontrar diferencias estadísticamente significativas para considerar que los grupos son equiparables y aplicar posteriormente pruebas estadísticas de inferencia convencionales (no multivariantes). Este es un error bastante extendido en la literatura $(9,24,25)$ que debe ser evitado. Así, aunque los PS no constituyen el único medio de ajuste disponible, en situaciones como la de este estudio, su rendimiento puede ser elevado.

\section{CONCLUSIÓN}

En el presente estudio no se ha podido demostrar que el grado de especialización del cirujano sea un factor determinante en el pronóstico de los pacientes sometidos a cirugía por cáncer de colon en nuestro Servicio de Cirugía. Más bien, lo que influyó en el resultado fueron los rasgos basales de los pacientes y del tumor. Por otra parte, este trabajo ha servido para dar muestra de la utilidad de los propensity scores en el ajuste de los resultados en estudios retrospectivos no aleatorizados, especialmente cuando el factor de estudio es poco frecuente, y de qué manera pueden cambiar dramáticamente el sentido de los resultados. Como se ha comentado, estos índices de propensión pueden ser utilizados en numerosas situaciones en investigación clínica, evitando sesgos producidos por la distribución no aleatoria de los pacientes en uno u otro grupo de estudio.

\section{BIBLIOGRAFÍA}

1. Begg CB, Cramer LD, Hoskins WJ, et al. Impact of hospital volume on operative mortality for major cancer surgery. JAMA 1998; 280: 1747-51.

2. Layne C. Does practice really make perfect? Ann Intern Med 2003; 139: 696-8.

3. García-Granero E. El factor cirujano y la calidad de la cirugía en el pronóstico del cáncer de recto. Implicaciones en la especialización y organización. Cir Esp 2006; 79: 75-7.
4. García-Granero E, Martí-Obiol R, Gómez-Barbadillo, et al. Impact of surgeon organization and specialization in rectal cancer outcome. Colorectal Dis 2001; 3: 179-84.

5. Harmon JW, Tang DG, Gordon TA, et al. Hospital volume can serve as a surrogate for surgeon volume for achieving excellent outcomes in colorectal resection. Ann Surg 1999; 230: 404-13.

6. Hermanek P, Weibelt H, Staimmer D, et al. Prognostic factors of rectum carcinoma -experience of the German Multicenter Sudy SGCRC. German Study Group Colo-Rectal carcinoma. Tumori 1991; 81: $60-4$.

7. Holm T, Johansson H, Cedermark B, et al. Influence of hospital- and surgeon-related factors on outcome after treatment of rectal cancer with or without preoperative radiotherapy. Br J Surg 1997; 84: 65763.

8. Kee F, Wilson, RH, Harper C, et al. Influence of hospital and clinician workload on survival from colorectal cancer: Cohort study. BMJ 1999; 318: 1381-5.

9. Luján J, Hernández Q, Valero G, et al. Influencia del factor cirujano en el tratamiento quirúrgico del cáncer de recto con quimiorradioterapia preoperatoria. Estudio comparativo. Cir Esp 2006; 79: 89-94.

10. McArdle CS, Hole DJ. Influence of volume and specialization on survival following surgery for colorectal cancer. Br J Surg 2004; 91: 610-7.

11. Mella J, Biffin A, Radcliffe AG, et al. Population-based audit of colorectal cancer management in two UK health regions. Br J Surg 1997; 84: $1731-6$.

12. Meyerhardt JA, Catalano PJ, Schrag D, et al. Association of hospital procedure volume and outcomes in patients with colon cancer at high risk for recurrence. Ann Intern Med 2003; 139: 649-57.

13. Parry JM, Collins S, Mathers J, et al. Influence of volume of work on the outcome of treatment for patients with colorectal cancer. $\mathrm{Br} \mathrm{J}$ Surg 1999; 86: 475-81.

14. Porter GA, Soskolne CL, Yakimets WW, et al. Surgeon-related factors and outcomes in rectal cancer. Ann Surg 1998; 227: 157-67.

15. Rosen L, Stasikk JJ, Reed JF, et al. Variations in colon and rectal surgical mortality. Comparison of specialties with a state-legislated database. Dis Colon Rectum 1996; 39: 129-35.

16. Schrag D, Cramer LD, Bach PB, et al. Influence of hospital procedure volume on outcomes following surgery for colon cancer. JAMA 2000; 284: 3028-35.

17. Schrag D, Panageas KS, Riedel E, et al. Hospital and surgeon procedure volume as predictors of outcome following rectal cancer resection. Ann Surg 2002; 236: 583-92.

18. Schrag D, Panageas KS, Riedel E, et al. Surgeon volume compared to hospital volume as a predictor of outcome following primary colon cancer resection. J Surg Oncol 2003; 83: 68-79.

19. Simunovic M, Rempel E, Thériault ME, et al. Influence of hospital characteristics on operative death and survival of patients after major cancer surgery in Ontario. Can J Surg 2006; 49: 251-8.

20. Smith JAE, King PM, Lane RHS, et al. Evidence of the effect of "specialization" on the management, surgical outcome and survival from colorectal cancer in Wessex. Br J Surg 2003; 90: 583-92.

21. Rosenbaum PR, Rubin DB. The central role of the propensity score in observational studies for causal effects. Biometrika 1983; 70: 41-55.

22. Adamina M, Guller U, Weber WP, et al. Propensity scores and the surgeon. Br J Surg 2006; 93: 389-94.

23. Newgard CD, Hedges JR, Arthur M, et al. Advanced statistics: The propensity score - a method for estimating treatment effect in observational research. Acad Emerg Med 2004; 44: 953-61.

24. Escrig-Sos J. Comparación de resultados quirúrgicos: un mínimo control, por favor. Cir Esp 2006; 80: 342-3.

25. Luján-Mompeán JA. Respuesta de los autores. Cir Esp 2006; 80: 340-4. 